\title{
Off-label use of medicines in neonates, infants, children, and adolescents: a joint policy statement by the European Academy of Paediatrics and the European society for Developmental Perinatal and Pediatric Pharmacology
}

\author{
Lenneke Schrier $^{1}$ (D) - Adamos Hadjipanayis ${ }^{2} \cdot$ Tom Stiris $^{3} \cdot$ Rob I Ross-Russell ${ }^{4} \cdot$ Arunas Valiulis $^{5} \cdot$ Mark A Turner $^{6}$. \\ Wei Zhao ${ }^{7} \cdot{\text { Pieter De } \text { Cock }^{8} \cdot \text { Saskia N de Wildt }}^{9} \cdot$ Karel Allegaert $^{10} \cdot$ John van den Anker $^{11,12}$
}

Received: 24 October 2019 / Revised: 18 December 2019 / Accepted: 20 December 2019

(C) Springer-Verlag GmbH Germany, part of Springer Nature 2020

\begin{abstract}
Health-care professionals who prescribe medicines have the professional duty to choose medicines that are in the best interest of their individual patient, irrespective if that patient is an adult or a child. However, the availability of medicines with an appropriate label for pediatric use is lagging behind those for adults, and even available pediatric drugs are sometimes not suitable to administer to children. Consequently, health-care professionals often have no other option than to prescribe off-label medicines to children. An important reason for use of off-label medicines is to improve access to (innovative) treatments or to address medical needs and preferences of patients, especially when no other options are available. However, off-label use of medicines is in general not supported by the same level of evidence as medicines licensed for pediatric use. This may result in increased uncertainty on efficacy as well as the risk for toxicity and other side effects. In addition, liability may also be of concern, counterbalanced by professional guidelines.

Conclusion: The purpose of this joint EAP/ESDPPP policy statement is to offer guidance for HCPs on when and how to prescribe off-label medicines to children and to provide recommendations for future European policy.
\end{abstract}

Keywords Off-label medicines $\cdot$ European guidance $\cdot$ Rational medicine use $\cdot$ Pediatrics

\section{List of abbreviations}

BNF-c British National Formulary for Children

CJEU Court of Justice of the European Union

EAP European Academy of Pediatrics

Communicated by Peter de Winter

Lenneke Schrier

L.Schrier-2@ prinsesmaximacentrum.nl

Adamos Hadjipanayis

adamos@paidiatros.com

Rob I Ross-Russell

robert.ross-russell@ntlworld.com

Arunas Valiulis

arunas.valiulis@mf.vu.lt

Mark A Turner

mark.turner@liverpool.ac.uk
ESDPPP European Society for Developmental Perinatal and Pediatric Pharmacology

GP General practitioner

HCP Health-care professional
Wei Zhao

zhao4wei2@hotmail.com

Pieter De Cock

pieter.decock@uzgent.be

Saskia N de Wildt

Saskia.deWildt@radboudmc.nl

Karel Allegaert

karel.allegaert@uzleuven.be

John van den Anker

jvandena@cnmc.org

Extended author information available on the last page of the article 
PICU Pediatric intensive care unit

PTLD Post-transplant lymphoproliferative disease

NICU Neonatal intensive care unit

NKFK Dutch Expertise Centre for Pharmacotherapy in Children

RTU Recommendations for use

SmPC Summary of product characteristics

\section{Introduction}

Health-care professionals (HCPs), in close collaboration with pharmacists, who prescribe medicines, have the professional duty to choose medicines that are in the best interest of their individual patient. However, the availability of medicines with an appropriate label for pediatric use is lagging behind those for adults, and available pediatric drugs are often not suitable to administer to children [1]. As a result, HCPs often have no other option than to prescribe medicines to children outside the approved conditions for age, therapeutic indication, dose recommendation, formulation, and/or route of administration (i.e., off-label use [2]) or to prescribe a drug which has not received a license for use in adults or children (i.e., unlicensed medicines use [2]). The practice of off-label prescribing of medicines to children is substantial, both in hospital care and primary health care [3]. A recent report on the setting in the European Union indicates that off-label use of medicines in children is still widespread. Off-label use varies among European countries, with 13-69\% of prescriptions being offlabel in the hospital setting and 2-100\% in primary care [4].

European guidelines on off-label use of medicines in children could greatly benefit children, parents, and their HCPs. Therefore, the purpose of this joint policy statement - endorsed by both councils of the European Academy of Pediatrics and the European Society for Developmental Perinatal and Pediatric Pharmacology - is to offer guidance for these HCPs and to provide recommendations for any future European policy.

\section{European views on off-label use of medicines}

According to recent survey findings among European stakeholders, off-label use of medicines is perceived to have both advantages and disadvantages. An important advantage is the accelerated access of patients to (innovative) treatments and the appropriate treatment of medical conditions in patients, when no other options are suitable. On the other hand, the issue of liability in case of negative consequences of offlabel use is a widely recognized concern as a formal assessment of safety and efficacy by the relevant authorities has not been performed. Furthermore, friction between national authorities and the pharmaceutical industry may occur if economic reasons are prevailing [4].

The development of medicines for children is complicated by a small and heterogeneous market and methodological and ethical requirements specific for pediatric trials [5]. As a result, many medicines are not labeled for use in children. In order to improve this situation, there have been a number of initiatives, including the European Pediatric Regulation. It has been estimated that more than $50 \%$ of medicines used in children have not been tested for the specific age group [6]. In addition, medicines labeled for use in children may underperform with respect to their ability to provide the recommended dose, the suitability of the dosage form, and the presence of potential harmful excipients [7]. For example, the availability of melting or chewable tablets, more likely taken by younger children, appears to be limited [1]. Typical therapeutic areas of offlabel use in children include - but are not limited to infectious diseases, cardiology, dermatology, pain treatment, alimentary tract and metabolism, the respiratory system, and the central nervous system [4]. The highest frequency of off-label use of medicines is seen in patients treated in the NICU, PICU, and children with oncologic diseases.

By using off-label medicines, prescribers, children, and their families have more treatment options to discuss in order to provide a treatment that one finds most appropriate for the needs of the individual patient. In addition, based on the newest available scientific evidence, (innovative) medicines (type of drug, dose, and/or indication) can be prescribed to patients at an earlier stage before the required regulatory approval has been finalized or adopted [4]. However, as off-label use of medicines are in general not supported by the same level of preclinical and clinical evidence as medicines licensed for pediatric use, this may result in increased uncertainty on efficacy as well as the risk for toxicity [7], and prescribers and patients have less information at their disposal to decide to choose (prescriber) or accept (patient) the off-label treatment [4]. Finally, the issue of liability may also be a concern, though often counterbalanced by available professional guidelines [4].

Off-label prescribing is not regulated by European law [9]. European legislation only regulates the marketing of medicines and not the way medicines are ultimately used in clinical practice, which is a national competence or is captured in paralegal statements or guidelines on good practice. Prescribing on-label or off-label medicines is a decision taken within the relationship between a patient and the prescriber. The professional setting (both legal and paralegal) does not limit the right of prescribers to prescribe medicines to "onlabel" prescriptions only, as this would in many cases lead to a conflict of professional duties. Therefore, in practice, at the national level, off-label use of medicines is often ethically and legally "accepted" under restrictions. 
Several European countries have adopted special statutory regulations for off-label use of medicines and have good practice or professional guidelines for use, including reimbursement decisions (Tables 1, 2, 3). These regulations or policy tools aim for improvement of knowledge regarding efficacy and safety of off-label use. This regulation to encourage pharmaceuticals to file a license extension and/or to create the opportunity to apply research results immediately in a licensed setting is counterbalanced by the uncertainties related to reimbursement practices. In countries where no policy tools are in place, the predominant argument is that off-label use of medicines is an issue that should be dealt with in the context of the relationship between prescriber and patient rather than at level of the regulatory or health-care system [4].

\section{European policy statement on prescribing off-label or unlicensed drugs to children}

Off-label use of medicines is subject to several conditions. According to the Court of Justice of the European Union (CJEU), off-label use should remain exceptional in order to preserve the practical effect of the licensing procedure for medicines. Prescribing off-label medicine (1) should be limited to individual situations justified by medical considerations, (2) should be under the responsibility of the prescriber, (3) presupposes that the medicine is necessary to address the needs of the patient, (4) should follow a full assessment and examination of the patient, and (5) should be decided on the basis of purely therapeutic considerations [4].

Table 1 National temporary recommendations for off-label use of medicines ${ }^{\mathrm{a}}$

\begin{tabular}{|c|c|c|}
\hline Country & When off-label prescribing? & How? \\
\hline Belgium & $\begin{array}{l}\text { The evaluation of the individual practitioner's choice } \\
\text { implies an evaluation of possible alternatives, including } \\
\text { alternative treatments, alternative medicinal treatments, } \\
\text { and alternatives to a treatment, but in essence, there is } \\
\text { "therapeutic freedom" }\end{array}$ & $\begin{array}{l}\text { After informed consent of the parents and patient and after } \\
\text { clinical examination }\end{array}$ \\
\hline France & If prescriber deems it necessary for patient, given scientific & Prescriber must justify choice \\
\hline \multirow[t]{4}{*}{ No. 2011-2012 act (RTU) } & knowledge and absence of available alternative treatment & Informed consent is required \\
\hline & & If medication is part of RTU: prescriber should mention this \\
\hline & $\begin{array}{l}\text { If medicine is part of "Recommendations for use (RTU)" } \\
\text { scheme. An RTU for off-label use can be issued by the } \\
\text { French Agency if certain criteria are met }\end{array}$ & $\begin{array}{l}\text { on prescription so that pharmacists can control } \\
\text { prescription in this context. The marketing authorization } \\
\text { holder should set up patient follow-up }\end{array}$ \\
\hline & & $\begin{array}{l}\text { RTU medicines are reimbursed by the national health } \\
\text { insurance }\end{array}$ \\
\hline Italy & \multirow{3}{*}{$\begin{array}{l}\text { If indication relates to therapeutic area with unmet medical } \\
\text { need, companies do not want to perform clinical trials for } \\
\text { given indication }\end{array}$} & \multirow{3}{*}{$\begin{array}{l}\text { Off-label use requires support of phase II } \\
\text { Informed consent is required; } \\
\text { Reimbursed if application of law } 648 / 96\end{array}$} \\
\hline National Law n. 94/98 (Di & & \\
\hline $\begin{array}{l}\text { Bella Law), 648/96 } \\
\text { National Law }\end{array}$ & & \\
\hline Spain & \multirow{4}{*}{$\begin{array}{l}\text { Off-label use has to be exceptional and only limited to those } \\
\text { situations in which no approved alternative exists, with } \\
\text { respect to any restriction of the conditions for prescribing } \\
\text { and dispensing established in the authorization and the } \\
\text { therapeutic protocol of the center }\end{array}$} & \multirow{4}{*}{$\begin{array}{l}\text { Prescriber has to justify need in the clinical history } \\
\text { Informed consent is required } \\
\text { Prescriber must notify adverse events } \\
\text { Prescriber must comply with established recommendations } \\
\text { and therapeutic protocols }\end{array}$} \\
\hline National Royal Decree No. & & \\
\hline $1015 / 2009$ & & \\
\hline & & \\
\hline Switzerland & \multirow{2}{*}{$\begin{array}{l}\text { If it is proven that there is no authorized or available } \\
\text { alternative medicine that is applicable and equivalent }\end{array}$} & \\
\hline $\begin{array}{l}\text { Federal law on medicinal } \\
\text { products and medical } \\
\text { devices, Art } 9 \text {, Art } 26\end{array}$ & & \\
\hline Hungary & \multirow{5}{*}{$\begin{array}{l}\text { (1) If treatment with licensed medicine is not possible or } \\
\text { unsuccessful } \\
\text { Or } \\
\text { (2) Access to licensed medicine is inhibited to an extent that } \\
\text { would likely delay treatment }\end{array}$} & Prescriber must ensure that: \\
\hline Art 25 of Act XCV (2005), & & Based on experimental evidence, medicine offers potential \\
\hline subsection 6 & & of successful treatment or improve/stabilize patient \\
\hline & & $\begin{array}{l}\text { condition; medicine is licensed for distribution in } \\
\text { Hungary or another country; prescriber is specialist in } \\
\text { specific therapeutic area; prescriber's request for use of } \\
\text { this medicine in specific patient has been granted by } \\
\text { government body for pharmaceuticals. }\end{array}$ \\
\hline & & $\begin{array}{l}\text { Risk/benefit balance of off-label medicine is better than that } \\
\text { of the licensed medicine; based on experimental } \\
\text { evidence, medicine offers potential of successful } \\
\text { treatment or improve/stabilize patient condition; the } \\
\text { SmPC of licensed medicine does not contain } \\
\text { contra-indication regarding requested unlicensed } \\
\text { indication }\end{array}$ \\
\hline
\end{tabular}


Table 2 National measures to regulate reimbursement of off-label medicines ${ }^{\mathrm{a}}$

\begin{tabular}{|c|c|c|}
\hline Country & When off-label prescribing? & How? \\
\hline Belgium & $\begin{array}{l}\text { There is a Special Solidarity Fund for individual patients, but } \\
\text { the fund has limited resources. This is because the Belgian } \\
\text { reimbursement regulation is based on a positive list of } \\
\text { reimbursed products. Consequently, off label can potentially } \\
\text { qualify for reimbursement, not necessary linked to an } \\
\text { indication or age category. However, the request for addition } \\
\text { of a medicinal product to the positive list is the sole } \\
\text { responsibility of the marketing authorization holder }\end{array}$ & \\
\hline Hungary & & $\begin{array}{l}\text { Case-by-case evaluation: decision to reimburse is taken based } \\
\text { upon circumstances (including existing alternatives and } \\
\text { reasons why these are not sufficient) and costs }\end{array}$ \\
\hline $\begin{array}{l}\text { Germany } \\
\text { Section } 92 \text { para (1) } \\
\quad \text { Nr. } 6 \text { SGB V }\end{array}$ & & $\begin{array}{l}\text { The German Agency decides if off-label medicines are } \\
\text { reimbursed based on a scientific evaluation by its off-label } \\
\text { expert commissions. } \\
\text { Costs should be refunded also if there are only weak references } \\
\text { for efficacy, on condition that the patient suffers from a } \\
\text { life-threatening condition and alternatives are missing }\end{array}$ \\
\hline $\begin{array}{l}\text { Greece } \\
\text { Official Gazette } \\
\quad 545 / \mathrm{B}^{\prime} / 01-03-2012 \\
\text { Law } 4316 / 2014\end{array}$ & & $\begin{array}{l}\text { Ministerial decree is required in special cases and according to } \\
\text { international bibliographic references } \\
\text { Off-label indications could be reimbursed if included in } \\
\text { therapeutic protocols approved by the Central Committee of } \\
\text { Health Council }\end{array}$ \\
\hline
\end{tabular}

HCPs who prescribe off-label medicines to children should comply with ethical and professional standards. Divergence in drug and social laws and institutional and professional rules across Europe may complicate a uniform approach to prescribing off-label medicines in children. Nevertheless, the European Academy of Pediatrics and the European Society for Developmental Perinatal and Pediatric Pharmacology strongly recommend that the following conditions are considered when prescribing off-label medicines to neonates, infants, children, or adolescents. We hereby suggest that this may serve as a checklist for good practice.

Condition \#1 All other options, including the use of medicines approved by the regulatory authorities, are unavailable, not tolerated, less optimal, too expensive, not reimbursable by insurance companies, or containing potentially harmful excipients.

Off-label use is not the same as off-knowledge use.

\section{Condition \#2 The prescriber is competent to prescribe off- label medicines in children.}

An off-label medicine can only be prescribed by someone who has prescribing skills (rational drug therapy) and is knowledgeable about off-label use of medicines in children [10]. As children differ from adults with regard to disease etiology, pharmacokinetic and pharmacodynamic factors, and formulation acceptance, the prescriber should have specific knowledge and experience in the field of pediatrics. Although most HCPs appear to be familiar with the practice of off-label prescribing, most are not aware that the medicines they prescribe are indeed off-label medicines [11]. As this may have consequences for the monitoring of efficacy and adverse events, it is crucial that the prescriber should be aware that he or she is prescribing the medicine in an off-label manner.

Condition \#3 Off-label prescription of the medicine is appropriate to meet the needs of the individual patient within the available resources.

There are several situations in which it may be necessary to prescribe a medicine in an off-label manner.

- There may be a medical need where there is no labeled medicine available [4], or the labeled formulation or dosage is not age-appropriate. For example, most medicines used in neonatology have not been tested for the appropriate age and weight group, and most doses have been extrapolated from adult and older children. Moreover, the risk of administration errors can be reduced if ageappropriate off-label drug formulations are prescribed. In addition, off-label use is the rule and not the exception in patients with rare diseases, like clobazam for epilepsy in Dravet syndrome.

- Certain medicines may be licensed, available, and suitable for use in children in one country, but not in another; for example, midazolam oral suspension that is available in Germany but not in Belgium, despite being licensed at the European level. 
Table 3 National guidance to professionals on off-label medicine use in children ${ }^{\mathrm{a}}$

\begin{tabular}{|c|c|c|}
\hline Country & When off-label prescribing? & How? \\
\hline Belgium & & $\begin{array}{l}\text { When a suitable tested and approved alternative is available, prescribing } \\
\text { physicians' liability may be at (increased) risk if safety issues arise. If an } \\
\text { adverse event arises through the use of that drug, the treating physician would } \\
\text { have the burden of proof to demonstrate that its use was performed as standard } \\
\text { of care. Key characteristics: usual or common practice, scientific basis, and } \\
\text { informed consent }\end{array}$ \\
\hline $\begin{array}{l}\text { Lithuania } \\
\text { Law of Pharmacy No. X-709 } \\
\quad \text { (2006) } \\
\text { Law of Patients' rights, }\end{array}$ & & $\begin{array}{l}\text { Off-label use of licensed medicines is possible based on the decision of a council } \\
\text { or group of clinical pharmacologists if applicable. In the outpatient setting, it is } \\
\text { possible to prescribe off-label and extemporaneous medicines based on } \\
\text { experience and personal decision }\end{array}$ \\
\hline
\end{tabular}

safety and compensation

of harm No. I-1562 (1996)

Sweden

SFS 2010:659 and SFS

2014:821

UK

Good practice in prescribing and managing medicines and devices, 2013

If there is sufficient scientific evidence and clinical experience to prescribe medicine, informed consent is required

Prescriber must be convinced that there is sufficient evidence or experience of using medicine to demonstrate its safety and efficacy; NICE publishes evidence summaries for off-label and unlicensed medicines

Prescriber has responsibility for prescribing the medicine; prescriber is responsible for overseeing patient's care, monitoring, and follow-up (or should ensure this is done by another suitable physician); prescriber must have clear, accurate, and legible record of prescribed medicines and reasons for prescribing medicine off-label; informed consent is required.

Pediatric:

Royal College of Paediatrics and Child Health (Rev 2, 2013) [24]:

Where available, an appropriate licensed preparation should be prescribed and supplied in preference to an unlicensed preparation.

In general, it is not necessary to take additional steps, beyond those taken when prescribing licensed medicines, to obtain consent of parents, caregivers, and child patients to prescribe or administer unlicensed medicines or licensed medicines for unlicensed applications (off-label) ${ }^{\mathrm{b}}$

The Netherlands Art 68 of Medicines Act
If relevant professional body has developed protocols or professional standards with regard to the specific off-label use If protocols or standards are under development, prescriber and pharmacist are required to consult each other.
Pediatric:

Dutch Pediatric Society (Rev 4, 2018) [26]: The Dutch Pediatric Society has accepted the National Pediatric Formulary as expert guideline

Off-label use is considered appropriate if there is a medical need (no registered medicine available or registered medicine is suboptimal for individual patient)

There should be a positive balance between expected efficacy and risks based on available literature and assessed within multidisciplinary setting

Informed consent is required unless off-label use is documented in National Pediatric Formulary or in professional guideline

The prescribing physician should inform parents/caregivers/child about the benefits and risks of off-label or unlicensed use of medicines

Off-label treatment with medicines should be regularly and rigorously monitored and adverse events should be reported nationally

Tables 1- $3^{\mathrm{a}}$ Based on information provided by EAP members and data included in $[4,27,30]$; ${ }^{\mathrm{b}}$ used definitions for off-label and unlicensed use may vary across countries and publications ${ }^{2}$. For example, in the UK, off-label use of drugs is referred to as "unlicensed use of licensed drugs". No data: countries not participating in EU study: e.g., Luxemburg, Norway, Switzerland, Iceland, Latvia, Poland, Romania, Croatia, Albania, Macedonia, Serbia, Bosnia, and Herzegovina.

- The licensed medicine may (no longer) be appropriate (has become obsolete), for example, due to the use of harmful excipients (like ethanol or propylene glycol), and an off-label medicine provides an appropriate alternative to use.

- An off-label medicine may address patient's needs better than the licensed medicine in cases when the licensed medicine is minimally effective or ineffective or causes unacceptable side effects, resulting in lower treatment adherence [4].

- Off-label use may be part of the professional treatment guideline [4], for example, in cases where product summaries have not been updated (yet) despite available evidence [12], such as aminoglycoside dosing in neonates and infants. This type of off-label use allows physicians to use existing medicines in an innovative way, when 
evidence exists but formal licensing for children has not taken place (yet) [4], as may be the case in pediatric oncology (e.g., rituximab for treatment of post-transplant lymphoproliferative disease (PTLD)).

\section{Condition \#4 The off-label prescription should be rational and clinically appropriate.}

As with the use of licensed medications, the prescriber must ensure the off-label prescription is appropriate, i.e., the benefit/risk balance should be deemed positive for the individual patient on the basis of the available evidence [4]. All medicines have associated risks of adverse events. In the case of on-label prescribing, the prescriber can rely on the medicine's evaluation by the competent authorities. In the case of off-label prescribing, the prescriber has to weigh the benefitrisk ratio. Off-label prescribing is considered appropriate if (1) it is justified by best available evidence, preferably based on professional guidelines supported by relevant societies (e.g., national pediatric and/or pharmacist societies); (2) it occurs within the context of a formal research protocol; or (3) it pertains to exceptional use, justified by individual clinical circumstances. If none of these justifications is present, its use is generally not recommended (adapted from [13]).

If off-label prescribing is considered appropriate, the prescriber will not automatically be liable for negative impacts on the patient's health, especially if the off-label use is mentioned in a professional guideline or formulary.

Rational pharmacotherapy requires that patients receive medicines appropriate to their clinical needs, in doses that meet their own requirements, for an adequate period of time, and at a reasonable cost to them and their community [14]. Formularies may be effective in improving rational pharmacotherapy in children [15]. In the UK and the Netherlands, HCPs prescribing medicines to children are guided by information in the British National Formulary for Children (BNFc) and the Dutch National Formulary for Children. In the Netherlands, the development of this open-access formulary has resulted in the revision of many consensus-based dose recommendations and ensured uniformity in prescribing habits in the Netherlands [16]. However, it is important to emphasize that these formularies have still knowledge gaps, like for optimal use of medicines in preterm neonates.

\section{Condition \#5 The patient and parents/caregivers should be informed and involved.}

When considering prescribing an off-label medicine, both children and their parents/caregivers should have all the appropriate information available, if possible, regarding that medicine.

There is limited published literature about the views of patients, parents, and health-care professionals regarding informed consent in off-label or unlicensed use of medicines in children. In general, most children [17], parents [11, 18], and citizens [19] feel that parents should be informed when a medicine is prescribed in an off-label or unlicensed manner, for example, to create alertness to potential side effects [17]. In addition, most older children feel that they also should be informed [17]. The literature from health-care professionals is more mixed: most health-care professionals in Northern Ireland [20] felt that parents should be informed, whereas hospital-based pediatricians in Scotland did not [21]. Knowledge about off-label or unlicensed use of medicines in children is low among the general public [19] and in different studies of parents of healthy and chronically ill children [11, 18]. A minority of hospital-based pediatricians [21] and of general practitioners [22] (GP) in Scotland informed a child's GP [21] or the parents [22]. Once parents knew that their children were prescribed off-label medicines, parents would ask for a licensed medicine $[18,19]$, or they would use the medicine with more caution [19]. The percentage of refusal of offlabel use was higher among parents of otherwise healthy children compared with parents of chronically ill children [23].

As summarized in Tables 1 and 3, several national policy tools indicate that patients should be informed and provide consent when off-label or unlicensed medicines are prescribed, although it is not always clear to what extent this consent differs from the regular consent procedure. The Royal College of Paediatrics and Child Health stated that when prescribing off-label medicines to children - it is not necessary to take additional steps to obtain consent of parents, caregivers, and children beyond those taken when prescribing licensed medicines [24]. This is in line with the policy statement issued by the American Academy of Pediatrics in 2014, which states that administration of off-label medicines in children does not warrant special consent if it is based on sound medical evidence. Obviously, if the off-label use is experimental as part of a study, then the patient (and/or parent) should be informed of its experimental status and has to provide the study related assent and/or consent [25]. European HCPs should know and abide by the appropriate informed consent laws in their respective countries.

\section{Condition \#6 The patient should be monitored for efficacy and adverse events.}

Similar to licensed medications but possibly with a heightened vigilance [8], the prescriber and pharmacist should ensure appropriate monitoring. Adverse events should be reported to the national pharmacovigilance system by HCPs but also by the families (parents and or children). In case where medicines are prescribed that have reasonable rationale for use but insufficient evidence to mitigate safety, efficacy, and costeffectiveness concerns, yet they are not part of clinical research ("innovate off-label use"), then outcomes should be evaluated prospectively, documented appropriately, and reported to all stakeholders (HCPs and patients) in a timely fashion. Regular review should occur to reduce the risk of 
continued use that is not efficacious or is unsafe [28]. In this way, this condition is true for any prescription, including offlabel or unlicensed use.

\section{Condition \#7 The prescriber should consider if off-label prescribing should be part of a clinical trial.}

Finally, in order to increase our knowledge about the efficacy and safety of all medicines used in children, prescribers should be informed about clinical trials involving off-label medication in which the patient could participate and inform the parents and - when applicable - also the children.

\section{Policy statement: recommendations}

Off-label and unlicensed prescription practices occur. In order to facilitate the clinical practice of appropriate, rational and safe prescribing of off-label medicines to individual children, the EAP and ESDPPP strongly recommend that:

- All HCPs prescribing medicines to neonates, children, or adolescents have access to reliable and up-to-date information (where possible) on the medicine they prescribe. A European pediatric formulary with the best evidence on dosing and safety information could be a useful tool for improving the rational use of medicines in children and adolescents [15]. The BNF-c and the Dutch formulary could serve as templates [16].

- Pediatric clinical pharmacologists/pediatricians/pediatric pharmacists should be actively involved/ consulted in decision-making processes by hospital, pharmacotherapeutics committees, and national health-care authorities.

- Enhanced safety monitoring and reporting by parents and caregivers should be promoted when off-label medicines are prescribed.

- Parents and patients but also the public should be educated about off-label and unlicensed use of medicines.

- Where off-label use of a medicine is common and evidencebased, it should be the shared responsibility of marketing authorization holder and the relevant regulatory authorities to take appropriate measures to address legal uncertainty and safety concerns, including updating of the SmPC.

- Health authorities and health insurances should support and thus reimburse therapeutic practices that are evidence-based or advocated by a respectable and responsible body of professional opinion, regardless of labelling status.

- Legislation should be adopted that aims to effectively stimulate research into off-label medicines, including publication of clinical trial data [29]), and facilitate the registration of off-label uses with a positive benefit-harm balance.

\section{Conclusion}

HCPs often have no other option than to prescribe medicines to children outside the approved conditions for age, therapeutic indication, dose recommendation, formulation, and/or route of administration. This EAP/ESDPPP policy statement is intended to offer practical guidance to these HCPs on when and how to prescribe off-label medicines to children. Several recommendations should be considered when prescribing offlabel medicines. This list of recommendations may serve as a checklist for good practice.

Acknowledgments Tjitske van der Zanden, BSc managing director of the Dutch Expertise Centre for Pharmacotherapy in Children (NKFK), is kindly thanked for her expertise and critical review of the manuscript.

Authors' contributions This paper was conceptualized by Lenneke Schrier and John van den Anker. The first draft of the manuscript was written by Lenneke Schrier, and all authors commented on previous versions of the manuscript. All authors made substantial contributions to this policy statement and revised it critically for important intellectual content. All authors read and approved the final manuscript to be published. All authors agree to be accountable for all aspects of the work in ensuring that questions related to the accuracy or integrity of any part of the work are appropriately investigated and resolved.

\section{Compliance with ethical standards}

Conflict of interest The authors declare that they have no conflict of interest.

Ethical approval and informed consent This article does not contain any studies with human participants or animals performed by any of the authors. Therefore, ethical approval or informed consent does not apply.

\section{References}

1. van Riet-Nales DA, de Jager KE, Schobben AF, Egberts TC, Rademaker CM (2011) The availability and age-appropriateness of medicines authorized for children in the Netherlands. Br J Clin Pharmacol 72:465-473

2. Neubert A, Wong IC, Bonifazi A et al (2008) Defining off-label and unlicensed use of medicines for children: results of a Delphi survey. Pharmacol Res 58:316-322

3. Kimland E, Odlind V (2012) Off-label drug use in pediatric patients. Clin Pharmacol Ther 91:796-801

4. Weda M, Hoebert J, Vervloet M, et al (2017) Study on off-label use of medicinal products in the European Union. https://nivel.nl/sites/ default/files/bestanden/Report_OFF_LABEL_Nivel-RIVMEPHA.pdf

5. Ivanovska V, Rademaker CM, van Dijk L, Mantel-Teeuwisse AK (2014) Pediatric drug formulations: a review of challenges and progress. Pediatrics 134:361-372

6. European Medicines Agency (2016). 10-year report to the European Commission. General report on the experience acquired as a result of the application of the Paediatric Regulation EMA/ $231225 / 2015$ 
7. Tuleu C, Breitzkreutz J (2013) Educational paper: formulationrelated issues in pediatric clinical pharmacology. Eur J Pediatr 172:717-720

8. Bellis JR, Kirkham JJ, Thiesen S et al (2013) Adverse drug reactions and off-label and unlicensed medicines in children: a nested case-control study of inpatients in a pediatric hospital. BMC Med $11: 238$

9. European Court of Justice, T-452/14 Laboratoires CTRS v Commission, paragraph 76. http://curia.europa.eu/juris/liste.jsf? num $=\mathrm{T}-452 / 14 \&$ language $=\mathrm{EN}$

10. Curriculum for common trunk training in paediatrics. Agreed by the general assembly of EAP in Brussels, 6 December 2014. http:// eapaediatrics.eu/wp-content/uploads/2015/12/Agreed-Commontrunk-curriculum-training-LAST1.pdf

11. Balan S, Hassali MA, Mak VS (2015) Awareness, knowledge and views of off-label prescribing in children: a systematic review. Br J Clin Pharmacol 80:1269-1280

12. Pandolfini C, Campi R, Clavenna A, Cazzato T, Bonati M (2005) Italian paediatricians and off-label prescriptions: loyal to regulatory or guideline standards? Acta Paediatr (Oslo, Norway : 1992 94: 753-757

13. Gazarian M, Kelly M, McPhee JR, Graudins LV, Ward RL, Campbell TJ (2006) Off-label use of medicines: consensus recommendations for evaluating appropriateness. Med J Aust 185:544548

14. World Health Organization (1985). The rational use of drugs. Report of the Conference of Experts Geneva

15. Bonati M, Pandolfini C (2004) Is it time for a European formulary of paediatric medicines? Arch Dis Child 89:890-891

16. van der Zanden TM, de Wildt SN, Liem Y, Offringa M, de Hoog M (2017) Developing a paediatric drug formulary for the Netherlands. Arch Dis Child 102:357-361

17. Mukattash T, Trew K, Hawwa AF, McElnay JC (2012) Children's views on unlicensed/off-label paediatric prescribing and paediatric clinical trials. Eur J Clin Pharmacol 68:141-148

18. Bang V, Mallad A, Kannan S, Bavdekar SB, Gogtay NJ, Thatte UM (2014) Awareness about and views of parents on the off-label drug use in children. Int J Risk Saf Med 26:61-70

19. Mukattash TL, Millership JS, Collier PS, McElnay JC (2008) Public awareness and views on unlicensed use of medicines in children. Br J Clin Pharmacol 66:838-845

20. Mukattash T, Hawwa AF, Trew K, McElnay JC (2011) Healthcare professional experiences and attitudes on unlicensed/off-label paediatric prescribing and paediatric clinical trials. Eur J Clin Pharmacol 67:449-461

21. McLay JS, Tanaka M, Ekins-Daukes S, Helms PJ (2006) A prospective questionnaire assessment of attitudes and experiences of off label prescribing among hospital based paediatricians. Arch Dis Child 91:584-587

22. Ekins-Daukes S, Helms PJ, Taylor MW, McLay JS (2005) Off-label prescribing to children: attitudes and experience of general practitioners. Br J Clin Pharmacol 60:145-149

23. Lenk C, Koch P, Zappel H, Wiesemann C (2009) Off-label, offlimits? Parental awareness and attitudes towards off-label use in paediatrics. Eur J Pediatr 168:1473-1478

24. The use of unlicensed medicines or licensed medicines for unlicensed applications in paediatric practice (2013). Policy statement produced by the joint RCPCH/NPPG Standing Committee on Medicines

25. Frattarelli DA, Galinkin JL, Green TP, Johnson TD, Neville KA, Paul IM, van den Anker J, American Academy of Pediatrics Committee on Drugs (2014) Off-label use of drugs in children. Pediatrics 133:563-567

26. Nederlandse Vereniging voor Kindergeneeskunde (2018). Policy statement of the Dutch expertise Centre for Pharmacotherapy in Children and the Dutch Paediatric Society on the prescription of off-label medicines to children

27. Lenk C, Duttge G (2014) Ethical and legal framework and regulation for off-label use: European perspective. Ther Clin Risk Manag 10:537-546

28. Ansani N, Sirio C, Smitherman T, Fedutes-Henderson B, Skledar S, Weber RJ, Zgheib N, Branch R (2006) Designing a strategy to promote safe, innovative off-label use of medications. Am J Med Qual 21:255-261

29. Schrier L, Illy K, Valiulis A, Wyder C, Stiris T (2018) EAP viewpoint on unpublished data from paediatric clinical trials. Eur J Pediatr 177:275-277

30. Vannieuwenhuysen C, Slegers P, Neyt M, Hulstaert F, Stordeur S, Cleemput I, Vinck I (2015) Towards a better managed off-label use of drugs. Health services research (HSR) Brussels: Belgian health care knowledge Centre (KCE). KCE reports 252. D/2015/10.273/ 82

Publisher's note Springer Nature remains neutral with regard to jurisdictional claims in published maps and institutional affiliations.

\section{Affiliations}

\section{Lenneke Schrier ${ }^{1}$ (D) - Adamos Hadjipanayis ${ }^{2} \cdot$ Tom Stiris $^{3} \cdot$ Rob I Ross-Russell $^{4} \cdot$ Arunas Valiulis $^{5} \cdot$ Mark A Turner $^{6}$. Wei Zhao ${ }^{7}$ - Pieter De Cock $^{8} \cdot$ Saskia N de Wildt ${ }^{9} \cdot$ Karel Allegaert $^{10} \cdot$ John van den Anker ${ }^{11,12}$}

1 European Academy of Paediatrics (EAP), Princess Maxima Centre for Pediatric Oncology, Utrecht, The Netherlands

2 EAP; Paediatric Department, Larnaca General Hospital, Larnaca, Cyprus; European University Medical School, Nicosia, Cyprus

3 EAP; Faculty of Medicine, University of Oslo, Norway; Neonatal Intensive Care Unit, Oslo University Hospital, Oslo, Norway

4 EAP; Department of Paediatrics, Addenbrookes Hospital, Cambridge, UK

5 EAP Institute of Clinical Medicine and Institute of Health Sciences, Vilnius University Medical Faculty, Vilnius, Lithuania
6 European Society for Developmental Perinatal and Paediatric Pharmacology (ESDPPP) Institute of Translational Medicine, University of Liverpool, United Kingdom; Centre for Women's Health Research, Liverpool Women's Hospital, Liverpool, UK

7 ESDPPP; School of Pharmaceutical Science, Shandong University, Jinan, China

8 ESDPPP; Department of Pharmacy, Ghent University Hospital, Belgium and Heymans Institute of Pharmacology, Ghent University, Ghent, Belgium 
9 ESDPPP; Department of Pharmacology and Toxicology, Radboud Institute for Health Sciences, Radboud University Medical Center, Nijmegen, The Netherlands; Intensive Care and Department of Paediatric Surgery, Erasmus MC-Sophia Children's Hospital, Rotterdam, The Netherlands

10 ESDPPP; Department of Development and Regeneration, KU Leuven, Leuven, Belgium; Department of Pharmaceutical and Pharmacological Sciences, KU Leuven, Leuven, Belgium;
Department of Clinical Pharmacy, Erasmus MC, Rotterdam, the Netherlands

11 ESDPPP; Children's National Hospital, Washington, DC, USA

12 Intensive Care and Department of Pediatric Surgery, Erasmus Medical Centre - Sophia Children's Hospital, University of Basel Children's Hospital, Basel, Switzerland,

Rotterdam, The Netherlands 